\title{
Numerical simulations of laser-excited magnetic Bloch oscillations
}

\author{
Sergey Shinkevich and Olav F. Syljuåsen \\ Department of Physics, University of Oslo, P. O. Box 1048 Blindern, N-0316 Oslo, Norway \\ (Received 23 October 2012; revised manuscript received 22 January 2013; published 4 February 2013)
}

\begin{abstract}
We propose to use lasers to excite magnetic Bloch oscillations in one-dimensional easy-axis ferromagnets at low temperatures. This proposal is investigated numerically in detail for material parameters relevant for $\mathrm{CoCl}_{2} \cdot 2 \mathrm{H}_{2} \mathrm{O}$.
\end{abstract}

DOI: 10.1103/PhysRevB.87.060401

PACS number(s): 75.78.-n, 75.40.Mg, 75.10.Pq, 78.47.D-

According to quantum mechanics a particle in a periodic potential will oscillate in response to a constant force. Such Bloch oscillations (BOs) were predicted in the early days of quantum mechanics, ${ }^{1,2}$ but have only recently been experimentally demonstrated in very clean semiconductor superlattices, ${ }^{3}$ in Bose-Einstein condensates, ${ }^{4}$ and in optical ${ }^{5}$ and ultrasonic ${ }^{6}$ superlattices.

In condensed-matter systems the particles need not be of the ordinary kind resembling electrons. In particular, an elementary particle in a one-dimensional anisotropic ferromagnet is a domain wall separating regions of up and down spins. Such domain walls can have a dispersion relation like that derived from a periodic potential, and in the presence of a uniform magnetic field these magnetic systems have been predicted to show BOs. ${ }^{7}$ In particular the blue crystalline material $\mathrm{CoCl}_{2} \cdot 2 \mathrm{H}_{2} \mathrm{O}$ and $\mathrm{CoNb}_{2} \mathrm{O}_{6}$ have been proposed as candidate materials. However, no BOs have been experimentally observed in these to date. ${ }^{8,9}$

In a recent paper ${ }^{10}$ we have revisited the material $\mathrm{CoCl}_{2}$. $2 \mathrm{H}_{2} \mathrm{O}$ and refined the predictions of Ref. 7 for detecting spectral signatures of BOs in neutron-scattering experiments, taking also into account extra interactions present in the material. While our result indicates that the spectral signatures of BOs can indeed be observed in neutron scattering, the signatures are relatively weak, at the $10 \%$ level of the total spectral weight at finite temperatures. This is a consequence of neutrons being a relatively weak probe as they cause only single spin-flip excitations.

We propose here a more direct way to excite BOs by keeping the material at low temperature and induce excitations using a short laser pulse. Upon turning off the laser pulse the magnetization of the material will continue oscillating at the Bloch frequency. It has been known since long ago ${ }^{11,12}$ that light in the far-infrared frequency range can induce magnetic excitations in $\mathrm{CoCl}_{2} \cdot 2 \mathrm{H}_{2} \mathrm{O}$, but no time dependence of the magnetization was studied there.

In this Rapid Communication we model the laser pulse as a time-dependent perturbation to the Hamiltonian and investigate its effects by solving the time-dependent Schrödinger equation numerically. We show that BOs can be generated this way, and give appropriate laser frequencies and pulse-duration times.

The magnetic properties of $\mathrm{CoCl}_{2} \cdot 2 \mathrm{H}_{2} \mathrm{O}$ are described by the spin-1/2 Hamiltonian ${ }^{11,13}$

$$
H=-\sum_{i}\left(J_{z} S_{i}^{z} S_{i+1}^{z}+h_{z} S_{i}^{z}\right)+H_{d},
$$

where $H_{d}$ denotes subdominant terms to be discussed below. The ferromagnetic coupling $J_{z}=36.5 \mathrm{~K}^{11}$ is the dominant term in the Hamiltonian. Alone it causes neighboring spins to align their spin $z$ components, thus the energy of an excited state depends on the number of antialigned spin neighbors: domain walls, where each domain wall costs an energy of $J_{z} / 2$. In the presence of an external magnetic field along the $z$ axis, $h_{z}$, the energy will also depend on the number of spins opposing the field, implying pairwise confinement of domain walls. Such a bound state of two domain walls separating $l$ overturned spins, known as a spin cluster excitation, ${ }^{11}$ or simply a domain, has an energy $J_{z}+h_{z} l$ above the groundstate energy.

The term $H_{d}$ describes additional couplings that partly give dynamics to the domain walls and partly induce more domain walls,

$H_{d}=-\sum_{i}\left[J_{a}\left(S_{i}^{+} S_{i+1}^{+}+S_{i}^{-} S_{i+1}^{-}\right)+J_{\perp}\left(S_{i}^{+} S_{i+1}^{-}+S_{i}^{-} S_{i+1}^{+}\right)\right]$,

where $J_{a}=3.8 \mathrm{~K}^{9}$ and $J_{\perp}=5.43 \mathrm{~K} .{ }^{9} S_{i}^{ \pm}=S_{i}^{x} \pm i S_{i}^{y}$ are the usual raising and lowering operators. The $J_{a}$ term can move a domain wall two lattice spacings, thus mixing states with even (or odd) $l$. Similarly $J_{\perp}$ gives kinetic energy to the $l=1$ domain state. Both of these terms can also induce new domainwall pairs. However, with $J_{z}$ being the dominant coupling, extra domain walls will be energetically costly, thus we will restrict our calculations to states having a small number of domain walls $N_{d w}$.

When restricting to $N_{d w} \leqslant 2, H$ can be diagonalized. The energy spectrum is $E_{n}=J_{z}+\mu_{n} h_{z}$, where $\mu_{n}$ is found by solving an equation involving a ratio of Bessel functions. ${ }^{10,14,15}$ For high energies $\mu_{n} \approx n$, an integer, thus the energy spectrum becomes equidistant, the so-called Wannier-Zeeman ladder (WZL). ${ }^{16}$ The corresponding energy eigenfunctions $\left|\psi_{n}\right\rangle$ are Bessel functions. ${ }^{10}$ From these one can construct a timedependent state $|\chi(t)\rangle=\sum_{n} a_{n} e^{-i E_{n} t}\left|\psi_{n}\right\rangle$. When this state is dominated by energy eigenstates with energies from the equidistant region, the time dependence of the magnetization $M_{z}(t)=\left\langle\chi(t)\left|\sum_{i} S_{i}^{z}\right| \chi(t)\right\rangle$ becomes

$$
M_{z}(t)=c-2 x_{0} \sum_{n} \operatorname{Re}\left(a_{n}^{*} a_{n+2} e^{-i \omega_{B} t}\right),
$$

where $c$ is a constant, $\omega_{B}=2 h_{z} / \hbar$ is the Bloch frequency, and $x_{0}=2 J_{a} / h_{z}$. Thus the $\mathrm{BO}$ amplitude is proportional to $4 J_{a} / h_{z}$ times a factor which depends on the probability amplitudes $a_{n}$ of the excited states. Our BO amplitude is a factor 2 larger 


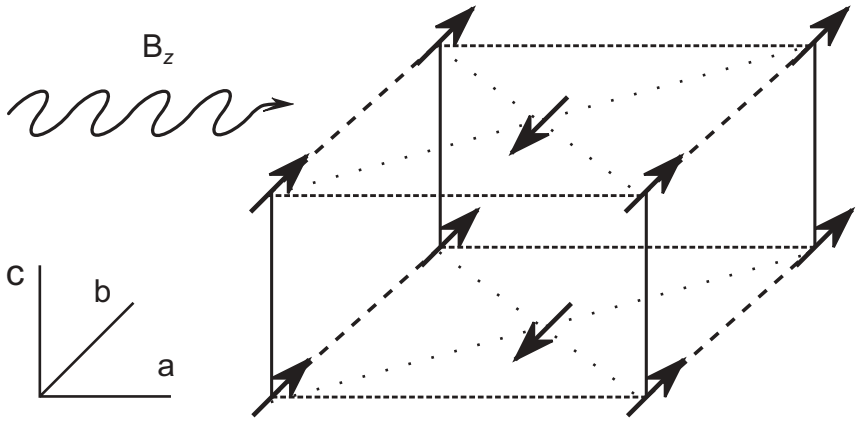

FIG. 1. In $\mathrm{CoCl}_{2} \cdot 2 \mathrm{H}_{2} \mathrm{O}$ the spin $z$ axis corresponds to the crystallographic $b$ axis. The strong ferromagnetic $J_{z}$ couples spins along the $c$ axis. A laser beam is shown propagating at normal incidence to the $b-c$ plane with magnetic-field polarization in the $b$ direction.

than obtained in Ref. 7 as we consider the size oscillations of a single domain having two domain walls, while they considered the motion of a single domain wall.

In order to populate the excited levels at very low temperatures, thus producing BOs, we propose here to use a laser with a wavelength in the far infrared. The Co electrons causing the magnetism are $d$-shell electrons thus having no electric dipole moment. ${ }^{17}$ We therefore model the laser as an extra time-dependent magnetic field which couples to the spins as

$$
H_{\mathrm{ext}}=-B_{0}^{z} \cos (\omega t) \sum_{i} S_{i}^{z}
$$

where $\omega$ is the laser frequency and $B_{0}^{z}$ is the laser magneticfield amplitude. We have here assumed a linearly polarized laser beam such that the magnetic field is along the Ising direction. This corresponds to the crystallographic $b$ direction in $\mathrm{CoCl}_{2} \cdot 2 \mathrm{H}_{2} \mathrm{O}$. Such a setup can be made by cleaving the crystals in the $b-c$ plane and directing the laser at normal incidence to this surface polarizing the laser beam such that the magnetic field points along the $b$ direction; see Fig. 1 . We have assumed the laser beam to be coherent along its front, and also through the crystal. The laser considered here has a wavelength of about $0.3 \mathrm{~mm}$ thus for this approximation to be good the crystal should be thinner than this. For thicker crystals there will be an additional phase shift associated with the depth.

The time-dependent Hamiltonian $H+H_{\text {ext }}$ can be treated numerically for large system sizes when restricting to states where $N_{d w} \leqslant 2$. This restriction implies that the energy gap between the ground state and any excited state will depend on the system size $N$. This can be understood by considering the perturbative energy correction from virtual processes involving the creation and destruction of an additional domain. As there are roughly $N$ places to insert the new domain, the energy correction will be proportional to $N$. When restricting to $N_{d w} \leqslant 2$ the ferromagnetic state receives this correction, but not the states having one domain, as their corrections come from the excluded $N_{d w} \geqslant 4$ sector. In order to make the energy gap intensive we redefine the coupling between the ferromagnetic and the $l=2, N_{d w}=2$ state in the Hamiltonian by dividing it by a factor $\sqrt{N} .{ }^{10}$ This effectively makes the correction to the ferromagnetic state independent of system size.

Starting in the ground state of $H$, the time-dependent Schrödinger equation is solved iteratively numerically with the laser field $H_{\text {ext }}$ present. In the iterations we keep the 300 lowest energy states of $H$ with zero momentum and $N_{d w} \leqslant 2$. The laser frequency $\omega$ is tuned such that $\omega=\left(E_{n}-E_{0}\right) / \hbar$ where $n$ corresponds to an energy level in the region where the spectrum is approximately equidistant. We choose $n=12$ corresponding to $E_{12}-E_{0} \approx 1.6 J_{z}$ for a static magnetic field $h_{z}=0.05 J_{z}$. With this we find essentially Rabi oscillations between the ground state and the $n=12$ excited level with a Rabi frequency $\omega_{R}=\left|B_{0}^{z} \alpha_{12}\right| / \hbar$, where $\alpha_{12}$ is the matrix element of $\sum_{i} S_{i}^{z}$ between the ground state and the $n=12$ excited state. Exciting the level $n=12$ alone does not give Bloch oscillations as one also needs to populate the levels with $n+2$ (or $n-2$ ); see Eq. (3). This can be achieved by using a large laser amplitude which causes off-resonant tunneling between the $n$ and the $n \pm 2$ levels. However, in order to get a sizable population of the $n \pm 2$ levels we have to use a laser amplitude as big as $B_{0}^{z}=0.2 J_{z}$, which, with a $g$ factor of $6.8,{ }^{11}$ corresponds to an electric-field amplitude of approximately $500 \mathrm{MV} / \mathrm{m}$. Experimentally this is too large as the dielectric breakdown field strength of most insulators is an order of magnitude less than this.

To achieve population of nearby levels with a smaller laser amplitude we propose instead to use two lasers, each in resonance with one of the two nearby levels. Specifically we use $B_{01}^{z}=0.01 J_{z}, \omega_{1}=\left(E_{12}-E_{0}\right) / \hbar$, and $B_{02}^{z}=0.03 J_{z}$, $\omega_{2}=\left(E_{14}-E_{0}\right) / \hbar$ in our simulation. The population of the different levels as a function of time after the lasers are turned on is shown in Fig. 2. Only even $n$ states are excited because the $S^{z}$ terms do not flip any spins. The black dot-dashed curve in Fig. 2 shows how the ground state is depleted. The minimum of the ground-state population coincides with the maxima of the population of levels $n=12$ and $n=14$, and occurs roughly at a time $2 \times 10^{4} \hbar / J_{z}$ for the parameters used here. Using smaller laser amplitudes this time gets larger proportional to the inverse laser amplitude.

We turn off $H_{\text {ext }}$ at the first maximum of the population curves, $\tau=20440 \hbar / J_{z}$. As the sum $\sum_{n} a_{n}^{*} a_{n+2}$ is dominated by the term $n=12$, the amplitude of the $\mathrm{BO}$ will be proportional to $\left|a_{12}^{*} a_{14}\right|$ which is shown in the lower inset of Fig. 2. It shows a beating pattern corresponding to the two frequencies $\omega_{1}$ and $\omega_{2}$. It may be difficult to turn off the laser when this quantity is maximal. However, this is not a major concern as the variation is small. The time-averaged value is approximately 0.48 .

Letting the system evolve further in time without $H_{\mathrm{ext}}$ produces the BOs shown in Fig. 3. As our simulation only allows single domain excitations we have plotted the relative size of the domain, measured by the expectation value of the number of spins opposing the field $N_{1 \downarrow}(t)=M_{z}(t)-N / 2$ divided by its time average $\bar{N}_{1 \downarrow} \cdot \bar{N}_{1 \downarrow} \approx n$ for excitation $E_{n}$. We see that the relative size of the domain oscillates between 0.7 and 1.3 corresponding to a size between 9.1 and 16.9 for $\bar{N}_{1 \downarrow} \approx(12+14) / 2=13$. Thus the amplitude is 3.9 which is close to the expected value $4 J_{a} / h_{z} \times 0.48=4.0$ from Eq. (3). Allowing a finite density $\rho$ of coherently oscillating domain states, the relative size of a single domain state 


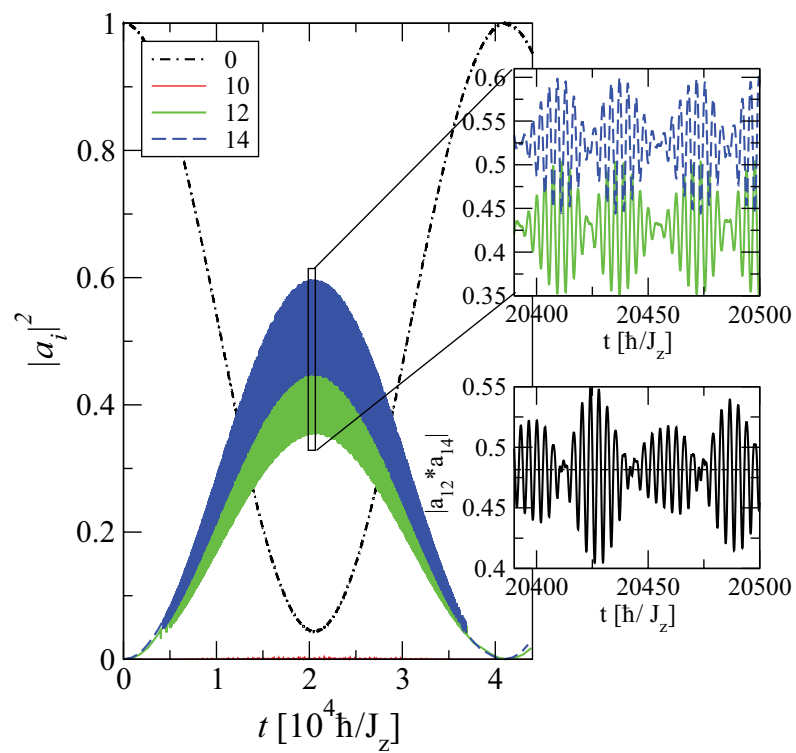

FIG. 2. (Color online) Population $\left|a_{i}\right|^{2}$ of selected energy levels $i$, indicated in the legend, as a function of time after turning on the two lasers. The laser frequencies and amplitudes are $\omega_{1}=\left(E_{12}-E_{0}\right) / \hbar$, $B_{01}^{z}=0.01 J_{z}$, and $\omega_{2}=\left(E_{14}-E_{0}\right) / \hbar, B_{02}^{z}=0.03 J_{z}$. The upperright panel shows a zoom in the boxed time region. The lower-right panel shows the time dependence of $\left|a_{12}^{*} a_{14}\right|$. The time-averaged value is shown as the horizontal dashed line. The time scale $\hbar / J_{z}$ is $0.2 \times 10^{-12} \mathrm{~s}$ for $\mathrm{CoCl}_{2} \cdot 2 \mathrm{H}_{2} \mathrm{O}$.

shown in Fig. 3 will be proportional to the experimentally relevant quantity, the time-dependent relative magnetization: $\left[M_{z}(t)-\bar{M}\right] / \bar{M}=\rho \bar{N}_{1 \downarrow} /\left(1 / 2-\rho \bar{N}_{1 \downarrow}\right)\left[N_{1 \downarrow}(t) / \bar{N}_{1 \downarrow}-1\right]$. Assuming noninteracting domains we estimate that the density of domains per unit length is $\rho=c\left(1-\left|a_{0}\right|^{2}\right) / \bar{N}_{1 \downarrow}$, where $c$ is a constant of the order unity. The lower panel of Fig. 3 shows the power spectrum of the BOs which reveals a single peak very close to $\omega_{B}=2 h_{z} / \hbar$. The small deviation
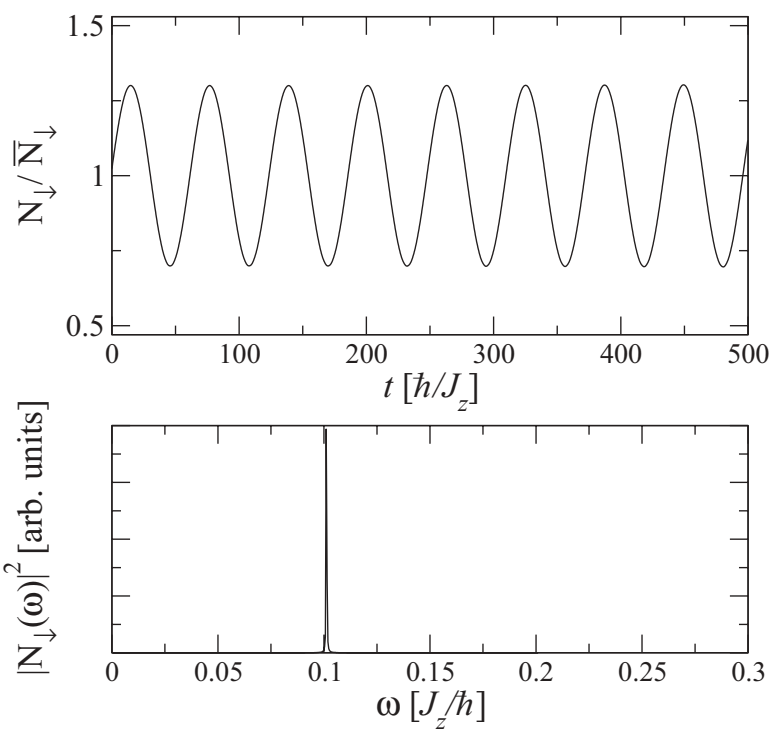

FIG. 3. Size oscillations of a domain excitation, measured as the number of down spins $N_{1 \downarrow} / \bar{N}_{1 \downarrow}$ vs time after the laser is switched off (upper panel), and its Fourier spectrum (lower panel).

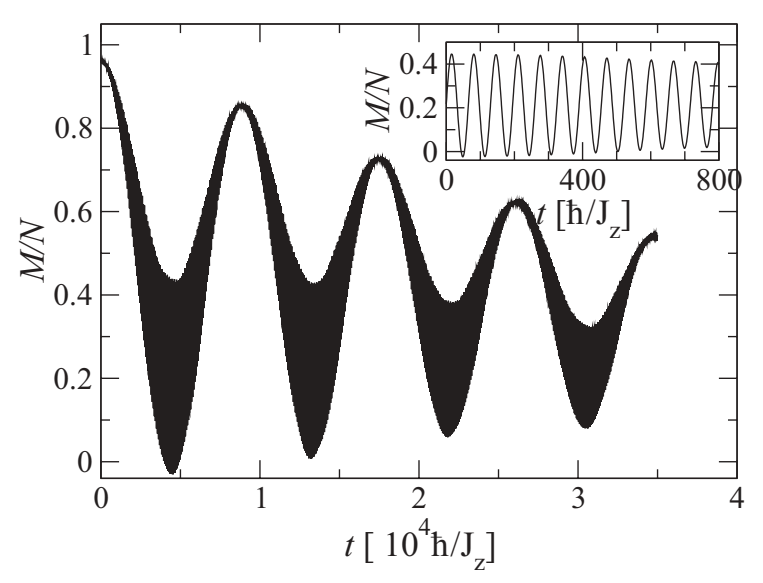

FIG. 4. Magnetization per site vs time for an interacting system in the presence of two laser beams, having amplitudes and frequencies as in Fig. 2 for $N=32$ and $N_{d w} \leqslant 6$. The inset shows the magnetization vs time after the two lasers have been switched off at $\tau=4420 \hbar / J_{z}$.

reflects the fact that the spectrum is not exactly the WZL at low energies. ${ }^{10}$

One can also change the laser polarization to have a component along the spin- $x$ direction. This will induce transitions between the even and odd $n$ states. Our simulations show BOs also in this case, but with $\omega_{B}=h_{z} / \hbar$, which corresponds to the energy spacing between adjacent even and odd levels.

The restriction to $N_{d w} \leqslant 2$ and the associated redefinition of the coupling to the ferromagnetic state can raise doubts about the validity of the matrix elements calculation, also it does not allow for any discussion of interactions between domains. We have therefore numerically investigated cases where we allow more domains without any redefinition of couplings. Computer performance restrictions let us consider $N_{d w} \leqslant 6$ for $N \leqslant 34$.

In order to address the effects of interactions between domains we do a time-dependent simulation of the magnetization of a $N=32$ system with $N_{d w} \leqslant 6$ in the presence of the two lasers. Figure 4 shows that the magnetization is rapidly varying, and has large-scale weakly damped oscillating behavior due to dephasing coming from populating other levels. We estimate the damping time scale to be roughly $5 \times 10^{4} \hbar / J_{z}$. We expect this time scale to decrease as the system size is increased. The time at which the magnetization envelope reaches its first minimum, $t_{m}=4420 \hbar / J_{z}$, is shorter than the time of maximal population in Fig. $2, \tau=20440 \hbar / J_{z}$, by a factor $k=t_{m} / \tau=1 / 4.6$. This is a reflection of the $\sqrt{N}$ in the matrix element connecting the ferromagnetic state to the single domain states, together with the near blocking by interactions and finite-size effects of states with more than one domain. ${ }^{18,19}$ For larger system sizes we expect that $k$ turns into $k=1 / \sqrt{R}$ where $R$ is the distance, in units of the lattice spacing, between domains beyond which the domain-domain interaction energy is smaller than $B_{01}^{z} \alpha_{12} \approx B_{02}^{z} \alpha_{14}$. This energy scale is $\sim 10^{-4} J_{z}$ for the parameters in Fig. 4 . When domains are closer than this the excitation of the second domain will be blocked by the presence of the first domain and will not participate in the resonance. On turning off the lasers at $t_{m}=4420 \hbar / J_{z}$ the magnetization oscillates at the Bloch frequency, shown in the inset of Fig. 4. 


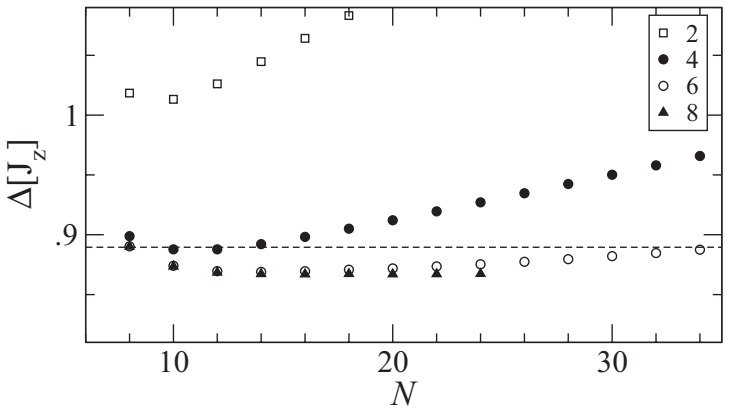

FIG. 5. Energy gap $\Delta$ to the first excited state vs system size $N$ for different $N_{d w}$. The legend specifies the maximum $N_{d w}$ included in the diagonalization. The dashed line shows the energy gap for $N_{d w} \leqslant 2$ with the $1 / \sqrt{N}$ redefinition of the coupling to the ferromagnetic state.

To see how energies are affected by allowing more domains we have plotted in Fig. 5 the $N$ dependence of the energy gap $\Delta$ to the first excited state. This decreases as higher domain-wall sectors are included. For comparison the $N_{d w} \leqslant 2$ result with redefined coupling to the ferromagnetic state is shown as the dashed line.

In order to identify the elementary domain excitations and the associated transition matrix elements, for the interacting system, we construct the approximate single domain creation operator $^{10}$

$$
a_{p, n}^{\dagger}=\sum_{l, j} \frac{e^{-i p\left(r_{j}+l / 2\right)}}{\sqrt{N}} J_{\left(l-\mu_{n}\right) / 2}\left(x_{0} \cos p\right) \Pi_{k=j}^{j+l-1} S_{k}^{+}
$$

with momentum $p=0$ and let it act on the ferromagnetic state. The sum is restricted to even values of $l$, and $J_{m}(x)$ is the Bessel function of the first kind of order $m$ with argument $x$. We then compute the overlap of this with the exact eigenstates of the $N_{d w} \leqslant 6$ system. For each value of $n$ we pick the state with maximum overlap. Figure 6(a) shows the energies of these
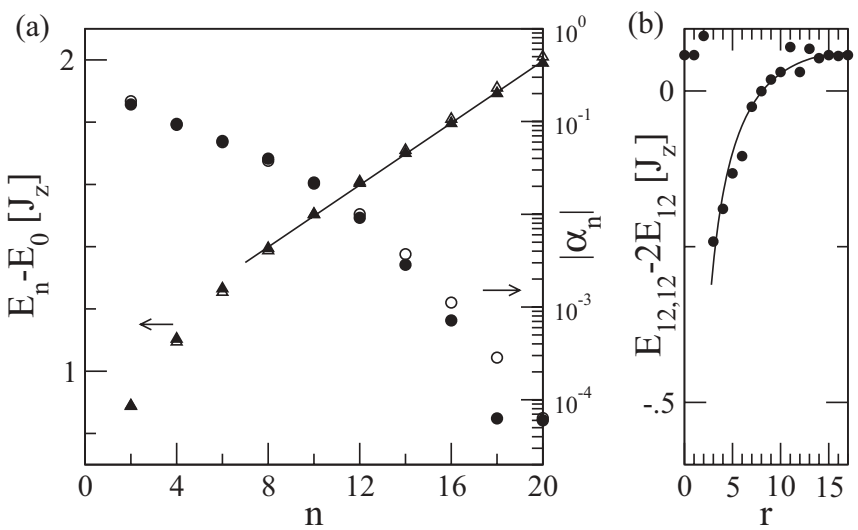

FIG. 6. (a) Excitation energy of elementary domain states vs $n$ (triangles). Also shown is $\left|\alpha_{n}\right|$ vs $n$ (circles) on a semilog scale. Filled symbols refer to $N_{d w} \leqslant 6$ and $N=34$, while open symbols are for $N_{d w} \leqslant 2$ with redefined coupling to the ferromagnetic state. (b) Interaction energy of two $n=12$ excitations vs separation distance $r$ for $N_{d w} \leqslant 6$ and $N=34$. states. We see that for high enough $n$ the energies become equidistant and agree well with what we found for $N_{d w} \leqslant 2$. The laser transition matrix element between these states and the ground state behaves as $\sqrt{N} \alpha_{n}$. The coefficients $\alpha_{n}$ are very close to those we found for $N_{d w} \leqslant 2$; see Fig. 6(a). Note that $\alpha_{n}$ drops very fast with increasing $n$.

Further insights about interactions can be gotten by identifying two-domain excitations. We construct approximate two-domain states as pairs with total momentum zero of two $n=12$ single domain states separated by a distance $r$, with creation operator $b_{r, n}^{\dagger}=\sum_{p} a_{p, n}^{\dagger} a_{-p, n}^{\dagger} e^{-i p r}$. We let this act on the ferromagnetic state, retain only the terms having four domain walls, and compute its overlap with the exact eigenstates of the $N_{d w} \leqslant 6$ system. The energy of the states with maximum overlap minus two times the single-particle excitation energy $E_{12}$ is shown in Fig. 6(b) as a function of $r$. We interpret this as the interaction energy of domains separated by $r$ lattice spacings. We find that the functional form $a-b[1 / r+1 /(N-r)]$ fits the results reasonably with $a=0.22 J_{z}$ and $b=1.37 J_{z}$. The positive $a$ is caused by the restriction on $N_{d w}$ which tends to overestimate energies in higher domain-wall sectors relative to those in lower sectors. This interaction energy causes an inhomogeneous broadening of the resonance frequency, and can lead to an upper limit on the density of domains excited by the laser together with an increased oscillation frequency due to interaction blocking effects, as seen in Fig. 4.

We conclude that it should be possible to excite magnetic BOs in $\mathrm{CoCl}_{2} \cdot 2 \mathrm{H}_{2} \mathrm{O}$ using two resonant lasers at low temperatures in a static magnetic field. On turning off the lasers after an excitation time $\tau$ the magnetization of $\mathrm{CoCl}_{2} \cdot 2 \mathrm{H}_{2} \mathrm{O}$ will oscillate at the Bloch frequency which is proportional to the static magnetic field and is $500 \mathrm{GHz}$ for $\mathrm{CoCl}_{2} \cdot 2 \mathrm{H}_{2} \mathrm{O}$ in a $0.4 \mathrm{~T}$ field. In order to generate the BOs one needs to populate at least two nearby levels in the WZL. Due to the relatively small laser transition matrix elements connecting the ground state to these levels we propose to use two large amplitude resonant lasers. It should be possible to use also resonant lasers with smaller amplitudes at the expense of longer excitation times, limited by the coherence time of the system. Collisions between domains can lead to dephasing behavior and associated decoherence. The domain excitations are heavy due to their flat dispersion, so we expect a significant effect only at a high density of excitations when neighboring domains are close together. We have performed a simulation taking into account the interaction between domains for a system of size $N=32$. From this we conclude that the interactions cause a blocking effect which speeds up the excitation process and that the dephasing time scale due to interactions is long compared with the excitation time for the parameters considered. Other effects might contribute to dephasing such as static impurities and interactions between chains. We have not studied these, but given the fact that the oscillations considered here are localized within a region of roughly 20 sites, we expect that the effects of static impurities are small for an impurity concentration of less than a few percent. In addition to $\mathrm{CoCl}_{2} \cdot 2 \mathrm{H}_{2} \mathrm{O}$ our simulations using the Hamiltonian and parameters from Ref. 20 also indicate that $\mathrm{BOs}$ in $\mathrm{CoNb}_{2} \mathrm{O}_{6}$ may be excited in a similar way. 
${ }^{1}$ F. Bloch, Z. Phys. 52, 555 (1929).

${ }^{2}$ C. Zener, Proc. R. Soc. London, Ser. A 145, 523 (1934).

${ }^{3}$ E. E. Mendez, F. Agullo-Rueda, and J. M. Hong, Phys. Rev. Lett. 60, 2426 (1988).

${ }^{4}$ M. Ben Dahan, E. Peik, J. Reichel, Y. Castin, and C. Salomon, Phys. Rev. Lett. 76, 4508 (1996).

${ }^{5}$ R. Sapienza, P. Costantino, D. Wiersma, M. Ghulinyan, C. J. Oton, and L. Pavesi, Phys. Rev. Lett. 91, 263902 (2003).

${ }^{6}$ H. Sanchis-Alepuz, Y. A. Kosevich, and J. Sánchez-Dehesa, Phys. Rev. Lett. 98, 134301 (2007).

${ }^{7}$ J. Kyriakidis and D. Loss, Phys. Rev. B 58, 5568 (1998).

${ }^{8}$ N. B. Christensen, K. Lefmann, I. Johannsen, and O. Jørgensen, Physica B 276, 784 (2000).

${ }^{9}$ W. Montfrooij, G. E. Granroth, D. G. Mandrus, and S. E. Nagler, Phys. Rev. B 64, 134426 (2001).
${ }^{10}$ S. Shinkevich and O. F. Syljuåsen, Phys. Rev. B 85, 104408 (2012).

${ }^{11}$ J. B. Torrance Jr. and M. Tinkham, Phys. Rev. 187, 587 (1969); 187, 595 (1969).

${ }^{12}$ D. F. Nicoli and M. Tinkham, Phys. Rev. B 9, 3126 (1974).

${ }^{13}$ H. C. Fogedby, Phys. Rev. B 5, 1941 (1972).

${ }^{14}$ H. C. Fogedby, Phys. Rev. B 10, 4000 (1974).

${ }^{15}$ S. B. Rutkevich, J. Stat. Mech. (2010) P07015.

${ }^{16}$ G. Wannier, Phys. Rev. 117, 432 (1960).

${ }^{17}$ W. J. L. Buyers, T. M. Holden, E. C. Svensson, R. A. Cowley, and M. T. Hutchings, J. Phys. C 4, 2139 (1971).

${ }^{18}$ M. D. Lukin, M. Fleischhauer, R. Cote, L. M. Duan, D. Jaksch, J. I. Cirac, and P. Zoller, Phys. Rev. Lett. 87, 037901 (2001).

${ }^{19}$ E. Urban, T. A. Johnson, T. Henage, L. Isenhower, D. D. Yavuz, T. G. Walker, and M. Saffman, Nat. Phys. 5, 110 (2009).

${ }^{20}$ J. A. Kjäll, F. Pollmann, and J. E. Moore, Phys. Rev. B 83, 020407(R) (2011). 